\title{
Anthrax: An Emerging Zoonotic Disease in Bangladesh
}

\author{
Be-Nazir Ahmed ${ }^{1}$, Yasmin Sultana ${ }^{2}$, DSM Fatema ${ }^{3}$, Khorshed Ara ${ }^{4}$, Nurjahan Begum ${ }^{5}$, S.M Mostanzid ${ }^{6}$, Shamim Jubayer ${ }^{7}$ \\ ${ }^{1}$ Institute of Epidemiology, Disease Control and Research (IEDCR); 2 Department of Physiology, National Institute of Cardiovascular Diseases (NICVD), Dhaka; 3 \\ EMO (General), DMCH, ${ }^{4}$ Department of Microbiology, Institute of Epidemiology, Disease Control and Research (IEDCR); ${ }^{5}$ Department of Obstetrics and Gynaecolog, \\ BSMMU, Dhaka; ${ }^{6}$ Department of Surgery, Faridpur Medical College, Faridpur; ${ }^{7}$ Department of Dental Public Health, University Dental College, Dhaka
}

\section{Introduction}

Anthrax has emerged as a zoonotic disease in Bangladesh during $2009-2010$. The human outbreaks were preceeded by animal outbreaks. The animal anthrax known as 'Torka' is believed to be enzootic for long in Bangladesh. The disease caused by the bacterium Bacillus anthracis is primarily a disease of herbivores and was one of the main causes of uncontrolled mortality in cattle, sheep, goats, horses and pigs worldwide until the development of an effective vaccine and advent of antibiotics. Although the organism has always been high on the list of potential agents with respect to biological warfare and bioterrorism, humans almost invariably contract the natural disease directly or indirectly from animals or animal products. ${ }^{1-5}$

\section{The organism}

Bacillus anthracis is a Gram-positive, rod-shaped nonmotile bacterium, the only obligate aerobic pathogen in the genus Bacillus. It has both vegetative and spore forms. Within low oxygen environment of the host, the organisms exclusively remain in the vegetative form. When conditions are not conducive to growth and multiplication, the vegetative forms start to form spores. Sporulation requires the presence of free oxygen. Spores are formed in cultures, in the soil, and in the tissues and exudates of dead animals. Spores are ellipsoidal or oval and are centrally located and are markedly resistant to biological extremes of heat, cold, $\mathrm{pH}$, desiccation, chemicals, irradiation and other such adverse conditions. ${ }^{3,6-8}$ The organisms grow well on most common laboratory media but for demonstration of characteristic colonial morphology, specimens should be inoculated on 5\% blood agar free of antibiotics. Maximal growth is obtained at $\mathrm{pH}$ 7.0-7.4 under aerobic conditions at $37^{\circ} \mathrm{C}$. The organisms produce large, raised, opaque, grayish white, plumose colonies $2-3 \mathrm{~mm}$ in

\author{
Correspondence: \\ Be-Nazir Ahmed \\ Professor of Microbiology and Chief Scientific Officer \\ Institute of Epidemiology Disease Control and Research (IEDCR \\ Mohakhali, Dhaka 1212 \\ E-mail : dbenazirahmed@yahoo.com, benazir1959@gmail.com
}

diameter, with an irregular, fringe like edge with no haemolysis. Tangled masses of long hair like curls can be seen with a colony microscope. The colony is membranous in consistency and emulsifies with difficulty. Though identification of typical virulent strains from clinical material is relatively simple but identification of strains from nonclinical material may be more difficult. $B$. cereus is easily mistaken for $B$. anthracis. The organism appears to be one of the most monomorphic species known, i.e. isolates from any source or geographical location are almost identical phenotypically and genotypically. In the past five years important progress has been achieved in determining phylogenetic relationships among isolates worldwide through multilocus variable number tandem repeat (VNTR) analysis (MLVA). ${ }^{3,9-10}$

\section{Global Situation}

Through successful national programmes, there has been a progressive global reduction in animal anthrax cases over the past three decades. The disease is now absent or only sporadic in the West Europe and the North America. Anthrax is hyperendemic in many countries of Africa with epizootic form in Chad and Ethiopia. In the Middle East, the disease is sporadic. Various levels of endemicity occur in central Asian countries, in western China and some other Southeast Asian countries. Anthrax is a severe problem in southern and eastern India. Humans are moderately resistant to anthrax and the incidence of anthrax in humans is low. Reported human: animal case ratios in a country reflect the economic condition, quality of surveillance, social traditions and dietary behaviour. Whereas in northern Europe, there has been one human infection per 10 livestock cases, in Africa and Asia, there can be some 10 human cases per one livestock infection. There are approximately 10-100 thousand human incidences annually throughout the world with significant numbers of cases in Chad, Ethiopia, Zambia, Zimbabwe and India. ${ }^{4,11-15}$

\section{Cycle of infection}

The spore is central to the cycle, although vegetative forms may also play a role in establishing infection. B. anthracis is not an invasive organism; it gains entry through breach of 
lining of surfaces. $\mathrm{LD}_{50} \mathrm{~s}$ ranges from $<10$ spores in susceptible herbivores to $>10^{7}$ in more resistant species. The $\mathrm{LD}_{50} \mathrm{~s}$ by inhalational or oral routes are much higher as compared with parenteral challenges in susceptible laboratory animals. Animals acquire anthrax by ingestion of spores while grazing or browsing. Flies appear to play an important role in explosive outbreaks. Inhalation within dust may be important on occasion. Direct animal to animal transmission is insignificant. ${ }^{4,16-17}$

\section{Incubation period (IP)}

Incubation period varies from few hours to even up to 60 days. For cutaneous anthrax, IP ranges from few hours to 3 weeks but most often it is 2 to 6 days; for gastrointestinal anthrax the average IP is 3-7 days. In inhalation anthrax, the median incubation period is 4 days, but may be up to 10 or 11 days. $^{4}$

\section{Pathogenesis}

Humans become infected in one of three ways percutaneous, inhalation and gastrointestinal. In cutaneous route, the organisms gain access through small abrasions or cuts and multiply locally with an inflammatory response. In inhalation anthrax, the organisms multiply in the lung, and are swept to the draining hilar lymph nodes leading to marked haemorrhagic necrosis. In ingestion anthrax, organisms are ingested with infected meat, with resultant invasion and ulceration of the gastrointestinal mucosa. From all three forms, invasion of blood stream and profound toxaemia may occur. Metastatic infections such as meningitis may complicate the primary process. Anthrax infection in humans provides permanent immunity and second attacks are rare. Though in general, the case fatality rate (CFR) due to anthrax has been reduced due to antibiotics but all three forms are potentially fatal if untreated and CFR is substantial in situation of use anthrax as biological weapon. ${ }^{18-22}$

\section{Clinical manifestation}

Cutaneous anthrax accounts for more than 95\% of human cases. It begins $2-5$ days after infection as a small papule resembling an insect bite developing within a few days into a vesicle filled with dark bluish black fluid. Rupture of the vesicle reveals a black eschar at the base with a very prominent inflammatory ring around the eschar. This is sometimes referred to as malignant pustule. The lesion is classically found on the hands, forearms or head and is painless. It is rarely found on the trunk or lower extremity. Generally, cutaneous lesions are single, but sometimes two or more lesions are present. The cutaneous form is more often self-limiting and with treatment, CFR is $<1 \%$ cases are fatal (Gold, 1967). 22-25

\section{Ingestion anthrax}

There are two clinical manifestations of ingestion anthrax - oropharyngeal anthrax and gastrointestinal anthrax. Oropharyngeal lesion is generally localized in the oral cavity covered with a grey pseudomembrane surrounded by extensive oedema. The main clinical features are sore throat, dysphagia and painful regional lymphadenopathy in the involved side of the neck. The gastrointestinal anthrax lesions are ulcerative and occur mostly in the ileum and caecum. The lesions may lead to haemorrhage, obstruction, perforation or any combination of these. Some cases are complicated with massive ascitis and this leads to shock and death. ${ }^{22,26,27}$

\section{Inhalational anthrax}

Inhalation anthrax occurs in persons who handle raw wool, hides or horsehair through inhalation of spores. The symptoms are typically those of respiratory tract infection with fever, malaise, mayalgia and an unproductive cough. Within several days, it rapidly progresses to a severe infection with marked respiratory distress and cyanosis, with the sudden worsening and death may occur within 24 hours. ${ }^{4}$

\section{Anthrax meningitis and sepsis}

Meningitis due to anthrax is a serious clinical development with symptoms of neck pain with or without flexion, headache, changes in mental state, vomiting and high-grade fever. A markedly elevated cerebrospinal fluid (CSF) pressure and the appearance of blood in the CSF are followed rapidly by disorientation, loss of consciousness and death. Anthrax sepsis develops after the lymphohaematogenous spread of $B$. anthracis from a primary lesion. Clinical features are high fever, toxaemia and shock followed by death. ${ }^{21,29-34}$

\section{Laboratory Diagnosis}

Microscopy with Gram stain and Polychrome methylene blue stain reveals respectively Gram positive rods and the capsule. On site anthrax specific antigen test by ICT may be done.

Culture of specimen obtained from skin lesion, sputum or blood will confirm the diagnosis.

Direct Polymerase Chain Reaction (PCR) on clinical specimens is regarded as an acceptable diagnostic procedure. Immunohistochemistry of pulmonary effusions or of bronchial biopsies is often proved to be valuable. In the case of patients who survive, antitoxin antibodies may be demonstrable in convalescent sera. Post mortem examination may reveal the capsulated $B$. anthracis usually visible in capsule-stained smears of these fluids, and should be easily isolated from them by bacteriological culture. Again, tests for toxin or PCR on these fluids would still be positive when treatment has rendered them smear- or culture-negative. ${ }^{4,} 26$, $27,36-42$

\section{Treatment of anthrax}

$B$. anthracis is quite responsive to antibiotic if administered early in the course of infection. Ciprofloxacin and doxycyline 
have received high profiles as primary treatment alternative in recent years. Doxycylcine has the disadvantage of poor penetration to the Central Nervous System (CNS). In severe life threatening cases, combination therapy with penicillin/ciprofloxacin and another antibiotic, preferably one which has good penetration into CNS are advisable. Clarithromycin, clindamycin, vancomycin or rifampicin are suggested for pulmonary anthrax and vancomycin or rifampicin is preferable for anthrax meningitis. B. anthracis is not susceptible to cephalosporin or trimethoprimsulfamethoxazole. Although susceptible to penicillin and ampicillin, due to presence of inducible beta-lactamase, these drugs are not recommended. , $^{43}$

\section{Prevention of Anthrax}

Control of human infection ultimately depends on control of the disease in animals. To cremate the carcass or to bury it deeply in lime to prevent soil contamination. Burning of the field may also be required. Active immunization is the only known method of preventing anthrax in herbivorous animals in areas where the pasture land is already contaminated with spores. Pasture's famous attenuated living anthrax vaccine was effective but difficult to maintain at a desired level of virulence. It has been superseded by a living spore vaccine derived from a nonencapsulated strain of B. anthracis (Sterne strain). The widespread use of a living spore vaccine in South Africa has reduced the incidence of anthrax in cattle of this area by more than $99 \%{ }^{4}$

\section{Human Immunization}

For the protection of humans in high-risk work situation, a nonliving vaccine consisting of aluminum hydroxideadsorbed supernatant material from fermentor cultures of a toxigenic but nonencapsulated strain of $B$. anthracis is used. The antigen appears to be effective but requires multiple doses over long periods of time. The cell free filtrate vaccine is available for veterinarians and others who work with livestock. Six inoculations over an 18-months period are $93 \%$ effective. Only four countries can produce the vaccine. In the USA, there is no vaccine for civilian use, but since 1998, all military personnel receive the cell-free filtrate vaccine. Prolonged antibiotic prophylaxis is only recommended to those exposed to deliberate use of anthrax spore. Antibiotic prophylaxis should not be used for naturally occurring anthrax. Use of personal protective equipment has to be encouraged. ${ }^{4}$

\section{Bangladesh situation}

Though there are not many reports, but anthrax in animal locally known as 'Torka' is believed to be enzootic in Bangladesh. Sixty two animal infections were recorded with 69\% deaths from Pabna milk shed areas during 1980-84. In another report, there were found to be 333 animal cases during 1989-96 from Bangladesh. A number of outbreaks of animal anthrax were reported during 2009-10 infecting 140 cattle and goat in different districts. The country has an estimated 23 million cattle, 1 million buffalo, 21 million goat and 3 million sheep. To prevent anthrax vaccination is practiced in the country since long. In 2009-2010, the total vaccine production was 38.29 million doses. Various reports indicate that the disease is more prevalent in areas-Pabna, Sirajganj and Tangail having greater cattle population. , $^{2,44}$

Like that of animal anthrax, reports for human anthrax are also lacking. So the true picture of anthrax is not available. As the disease is thought to be enzootic and in agricultural setting, humans always get infection from infected animal, so anthrax is also likely to be prevailing in the country in endemic form. Samad and Haque (1986) reported 27 human cases of anthrax during 1980-84 and a research team of Institute of Epidemiology, Disease Control and Research (IEDCR) detected 19 cases out 624 tannery workers of Dhaka city in 1997. In 2009 and 2010, there were twenty nine human anthrax outbreaks with 706 outbreaks in 12 districts. $2,4,44$

Table I : Reported findings of anthrax in animal and humans in Bangladesh

\begin{tabular}{ccc}
\hline Year & Animal case & Human case \\
\hline 1980 & 6 & 00 \\
1981 & 7 & 00 \\
1982 & 16 & 17 \\
1983 & 21 & 10 \\
1984 & 12 & - \\
$1989-96$ & 333 & - \\
1997 & 00 & 19 \\
October 2009- June 2010 & 55 & 607 \\
August 2010- October 2010 & 140 & 725 \\
Total & 450 & \\
\hline
\end{tabular}

All the human cases were cutaneous anthrax and no inhalation or ingestion anthrax was ever reported from Bangladesh. The skin lesion was characterized by the presence of papule and/or vesicle, ulcer, erythema, central black eschar, surrounding oedema and tenderness. The lesions were mostly distributed on the upper limbs $(75 \%)$, but were also present on lower limbs, face, chest, back, neck and scalp. Almost all of the cases with cutaneous lesion (97\%) had slaughtered sick cattle or goats, or handled raw meat or were present at the slaughtering site. ${ }^{2}$

Microbiologists of the IEDCR identified Bacillus anthracis from three vesicular swab samples tested from the first outbreak and from eight out of 12 vesicular samples tested from the fourth outbreak and did the sensitivity test. All the isolates were sensitive to ciprofloxacin and doxycycline, which were identified as the drug of choice for the cases. ${ }^{2}$ Centre for Disease Control (CDC), USA isolated B. anthracis from swabs of two patients. Multiple-locus variable-number 
tandem repeat analysis (MLVA) was performed at the CDC on the animal and human isolates and confirmed that both were infected by isolates of the same genotype. $B$. anthracis was also detected in three tissue biopsies by Immunohistochemistry. It was also detected from four vesicular swab smears by M'Fadyean staining. Fifteen of the 26 case-patients, who had acute and convalescent sera tested at $\mathrm{CDC}$, exhibited a four-fold increase in anti-PA (protective antigen) titre. ${ }^{2}$

\section{Conclusion}

Anthrax is rare in humans but occur among cattle in many parts of the world. B. anthracis remains in two forms: vegetative and spore. Cattle get infection exclusively with spore form. Human gets infection both in spore and vegetative forms. Human gets infection from three sources agricultural setting, industrial setting and deliberate use (bioterrorism). Human infection occurs in three forms cutaneous, intestinal and pulmonary. Cutaneous infection is the most common (>95\%). It can be caused by vegetative form or spore form and the organism enters through breached skin. The clinical diagnosis is made with epilink, characteristic ulcer, microscopy and culture. For uncomplicated anthrax, ciprofloxacin is the drug of choice. Prevention of human infection is based on prevention of anthrax in cattle through vaccination, avoidance of slaughtering sick cattle and use of PPE during handling of carcass.

\section{References}

1. Sterne M. The effects of different carbon dioxide concentrations on the growth of virulent anthrax strains. Pathogenicity and immunity tests on guineapigs and sheep with anthrax variants derived from virulent strains. Onderstepoort Journal of Veterinary Science and Animal Industry, 1937b; 9:49-67.

2. Chakraborty A, Uddin SK, Hasnat MA, Parveen S, Islam MS, Mikolon A et al. Anthrax outbreaks in Bangladesh, 2009 - 2010 (Personal communication)

3. Joklik WK, Willett HP, Amos DB, Wilfert CM. Zinsser Microbiology, 20 ${ }^{\text {th }}$ Edition, Appleton \& Lange, Norwalk, Connecticut. 1992; 615-20

4. WHO anthrax guidelines. Anthrax in humans and animals, $4^{\text {th }}$ Edition, WHO 2008; 1

5. Ahmed B, Sultana Y, Haque MM, Karim MR, Rahman D, Bacillus anthracis: an agent of biological weapon. JOPSOM 2001; 20(2): 45-50.

6. Titball RW, turnbull PC, Hhutson RA. The monitoring and detection of Bacillus anthracis in the environment. Society for Applied Bacteriology Symposium Series, 1991; 20:9S-18S.195

7. Quinn CP, turnbull PC . Anthrax. in: Collier L et al., eds. Topley and Wilson's microbiology and microbial infections. ninth ed. Vol. 3. London, Arnold, 1998; (799-818).

8. de Vos V, Turnbull PC . Anthrax. in: Coetzer JA, Thomson Gr,
Tustin RC, eds. Infectious diseases of livestock, with special reference to Southern Africa, 2nd ed. 2004.

9. Keim $\mathrm{P}$ et al. Multiple-locus variable-number tandem repeat analysis reveals genetic relationships within Bacillus anthracis. Journal of Bacteriology, 2000; 182:2928-36.

10. Kobuch WE et al. A clinical and epidemiological study of 621 patients with anthrax in western Zimbabwe. Salisbury Medical Bulletin, 1990; 68S:34-8.

11. Turner M. Anthrax in humans in Zimbabwe. Central African J. Med. 1980; 26:160-1.

12. Keim P, Smith KL. Bacillus anthracis evolution and epidemiology. in: Koehler tM, ed. Anthrax. Current Topics in Microbiology and Immunology, 2002; 271:23-32.

13. Pugh Ao, davies JC. human anthrax in Zimbabwe. Salisbury Med. Bull. 1990; 68S:32-3.

14. Heyworth B. Anthrax in the Gambia: an epidemiological study. Brit. Med. J. 1975; 4:79-82.

15. Davies JC. A major epidemic of anthrax in Zimbabwe. 1. the spread of the epidemic in Matabeleland, and Mashonaland Provinces during the period November 1978 to October 1980. Central African J. Med. 1982; 28:291-8.

16. Druett HA et al. Studies on respiratory infection with anthrax spores. 1. the infuence of particle size on respiratory infection with anthrax spores. J. Hyg. 1953; 51:359-71.

17. Watson A, Keir D. information on which to base assessments of risk from environments contaminated with anthrax spores. Epid. and Infect. 1994; 113: 479-90.

18. Abramova FA et al. Pathology of inhalational anthrax in 42 cases from the Sverdlovsk outbreak of 1979. Proceedings of the National Academy of Sciences, 1993; 90:2291-4.

19. Meselson M et al. the Sverdlovsk anthrax outbreak of 1979. Science, 1994; 266:1202-8.

20. Gumbel P. Sverdlosk. What really happened? Wall Street Journal, 1991; october 21, 22, 23.

21. Jernigan JA. Bioterrorism-related inhalational anthrax: the frst 10 cases reported in the united States. Emerging Infectious Diseases, 2001; 7:933-44.

22. Inglesby TV. Anthrax as a biological weapon, 2002. updated recommendations for management. J Am. Med. Association, 2002; 287: 2236-52.187

23. Jernigan DB. Investigation of bioterrorismrelated anthrax, united States, 2001: epidemiologic fndings. Emerg. Infect. Dis. 2002; 8:1019-28.

24. Phillips M. Cutaneous anthrax associated with bioterrorism, United States, 2001. Proceedings of $52^{\text {nd }}$ epidemic intelligence Service Conference.

25. Gold H. Treatment of anthrax. Federation Proceedings, 1967; $26: 1563-8$.

26. Doganay M, Almaç A, Hanagasi R. Primary throat anthrax. Scand. J. Infect. Dis. 1986; 18: 415-9.

27. Sirisanthana T. Outbreak of oral-oropharyngeal anthrax: an 
unusual manifestation of human infection with Bacillus anthracis. Am. J. Trop. Med. Hyg. 1984; 33:144-50.

28. Sirisanthana T, Brown AE. Anthrax of the gastrointestinal tract. Emerg. Infect. Dis. 2002; 8:649-51.

29. Levy LM. Anthrax meningitis in Zimbabwe. Central African J. Med. 1981;27:101-4.

30. Koshi G. Anthrax meningitis: a rare clinical entity. Journal of the Assoc. Phys. India, 1981; 29:59-62.

31. Lalitha MK. Unusual forms of anthrax - a clinical problem. Salisbury Med. Bull. 1990; 68S:38-40.

32. Lalitha MK. Anthrax - a continuing problem in Southern india. Salisbury Med. Bull. 1996; 87S:14-5.

33. George S. An outbreak of anthrax meningoencephalitis. Transactions of the Royal Society Trop. Med. Hyg. 1994; 88:206-7.

34. Kanungo R. Problem of timely diagnosis in anthrax meningitis. Journal of the Assoc, Phys. India, 2002; 50:913-5.

35. Ellerbrok H. rapid and sensitive identifcation of pathogenic and a pathogenic Bacillus anthracis by real-time PCR. FEMS Microbiology Letters, 2002; 214:51-9.

36. Shieh WJ. the critical role of pathology in the investigation of bioterrorism-related cutaneous anthrax. Am. J. Path. 2003; 163:1901-10.

37. Navacharoen N. oropharyngeal anthrax. J. Laryngology Otology, 1985; 99:1293-5.

38. Onerci M, Ergin NT. [oropharyngeal anthrax]. Laryngo-RhinoOtologie, 1993; 72:350-1. (in German, summary in english.)

39. Beatty ME. Gastrointestinal anthrax. Review of the literature. Arch. Int. Med. 2003; 163:2527-31.

40. Guarner J. Pathology and pathogenesis of bioterrorism-related inhalational anthrax. Am. J. Path. 2003; 163:701-9.

41. Pommerville JC. Alcamo's Fundamentals of Microbiology, 7th Edition, Jone and Bartlett Publishers, Boston, Toronto, London, Singapore. 2004; 344-346

42. Nelson KE, Williams CM, Graham NMH. Infectious Disease Epidemiology Theory and Practice. Aspen Publication Maryland, USA. 2001; 21-22, 313-314.

43. Boon NA, Colledge NR, Walker BR, Hunter JAA. Davidson's Principles and Practice of Medicine, 20th Edition, Churchil Livingstone Elsevier, Nodia, India. 2006;332-333, 341

44. Samad MA, Hoque ME. Anthrax in man and cattle in Bangladesh. Journal of Tropical medicine and Hygiene 1986:89, 43-45 